\title{
Spin injection across a hybrid heterojunction: Theoretical understanding and
} experimental approach (invited)

Hu, C.M.; Nitta, J.; Jensen, Ane; Hansen, Jørn Bindslev; Takayanagi, H.; Matsuyama, T.; Heitmann, D.; Merkt, U.

Published in:

Journal of Applied Physics

Link to article, DOI:

$10.1063 / 1.1447282$

Publication date:

2002

Document Version

Publisher's PDF, also known as Version of record

Link back to DTU Orbit

Citation (APA):

Hu, C. M., Nitta, J., Jensen, A., Hansen, J. B., Takayanagi, H., Matsuyama, T., Heitmann, D., \& Merkt, U. (2002). Spin injection across a hybrid heterojunction: Theoretical understanding and experimental approach (invited). Journal of Applied Physics, 91(10), 7251-7255. https://doi.org/10.1063/1.1447282

\section{General rights}

Copyright and moral rights for the publications made accessible in the public portal are retained by the authors and/or other copyright owners and it is a condition of accessing publications that users recognise and abide by the legal requirements associated with these rights.

- Users may download and print one copy of any publication from the public portal for the purpose of private study or research.

- You may not further distribute the material or use it for any profit-making activity or commercial gain

- You may freely distribute the URL identifying the publication in the public portal 


\title{
Spin injection across a hybrid heterojunction: Theoretical understanding and experimental approach (invited)
}

\author{
C.-M. $\mathrm{Hu}^{\mathrm{a})}$ \\ Institut für Angewandte Physik und Zentrum für Mikrostrukturforschung, Universität Hamburg, \\ Jungiusstrasse 11, 20355 Hamburg, Germany and NTT Basic Research Laboratories, 3-1 Wakamiya, \\ Morinosato, Atsugi-shi, Kanagawa 243-0198, Japan \\ J. Nitta \\ NTT Basic Research Laboratories, 3-1 Wakamiya, Morinosato, Atsugi-shi, Kanagawa 243-0198, Japan \\ A. Jensen and J. B. Hansen \\ Department of Physics, Technical University of Denmark, DK-2800 Lyngby, Denmark \\ H. Takayanagi \\ NTT Basic Research Laboratory, 3-1 Wakamiya, Morinosato, Atsugi-shi, Kanagawa 243-0198, Japan \\ T. Matsuyama, D. Heitmann, and U. Merkt \\ Institut für Angewandte Physik und Zentrum für Mikrostrukturforschung, Universität Hamburg, \\ Jungiusstrasse 11, 20355 Hamburg, Germany
}

\begin{abstract}
Spin injection across a hybrid ferromagnet/semiconductor junction has proven to be difficult, unlike in an all-metal junction used in giant magnetoresistance devices. The difference responsible is highlighted in a simple model. We perform spin-injection-detection experiments on devices with two ferromagnetic contacts on a two-dimensional electron gas confined in an InAs quantum well. We demonstrate that spin injection allows the hybrid device to combine both the advantage of the ferromagnet as well as that of the semiconductor. (C) 2002 American Institute of Physics.
\end{abstract}

[DOI: $10.1063 / 1.1447282]$

\section{INTRODUCTION}

The magnetoresistance (MR) effect caused by spin injection across a heterojunction was first demonstrated by Johnson and Silsbee ${ }^{1}$ in 1985, three years before the discovery of the giant MR (GMR) effect that revolutionized the sensor and storage industry. ${ }^{2}$ Now it is well known that by connecting two ferromagnetic (FM) metal electrodes with a nonmagnetic metal conductor [Fig. 1(a)], the resistance between the two FM electrodes can be modulated by changing their relative direction of magnetization. The simplest device uses two FM electrodes with different coercive fields, so that by sweeping an external applied magnetic field $B$, their relative direction of magnetization switches between a parallel and an antiparallel configuration [Fig. 1(b)], thus resulting in a change in resistance [Fig. 1(c)]. By improving the device geometry and combinations of materials, the normalized resistance change in such all-metal devices has been driven up to as high as $20 \%$ at ambient temperature to date. ${ }^{2}$ A new idea of great interest now is to replace the nonmagnetic metal conductor with a two-dimensional electron gas (2DEG) confined in semiconductors. A 2DEG, despite its

a) Author to whom correspondence should be addressed; electronic mail: chu@physnet.uni-hamburg.de smaller carrier density compared to that of a metal, is a metallic system with conductivity comparable to metals due to its much higher mobility. The hope of incorporating magnetic properties with mainstream semiconductor functionalities like gating, ${ }^{3}$ optical switching ${ }^{4}$ and ballistic transport ${ }^{5}$ in hybrid devices is invigorating to a flourishing new field of spintronics. ${ }^{6}$

The bottleneck in developing such hybrid devices turns out to be spin injection across the FM/2DEG interface. Despite great effort, ${ }^{7-12}$ the results achieved are rather controversial. Instead of the expected hysteretic magnetoresistance shown in Fig. 1(c), complicated features that differ from sample to sample are observed in most experiments, leaving plenty of room to argue or dispute and with different interpretations. The normalized resistance changes are mostly extremely small, below $1 \%$, in contrast to the reproducible and larger effect in all-metal GMR devices. ${ }^{2}$ After a major theoretical breakthrough discovered a basic obstacle for spin injection due to material mismatch, ${ }^{13}$ a serious question has been raised as to whether spin injection across a hybrid junction is measurable at all.

\section{THEORETICAL UNDERSTANDING}

Recent theoretical progress brings positive answers to this question. ${ }^{14-21}$ Using different approaches, most of these 
( a )

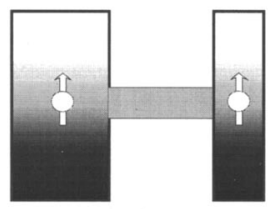

तB

( b )

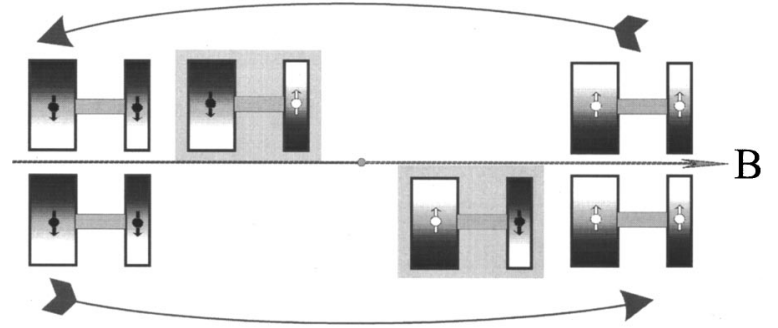

(c)

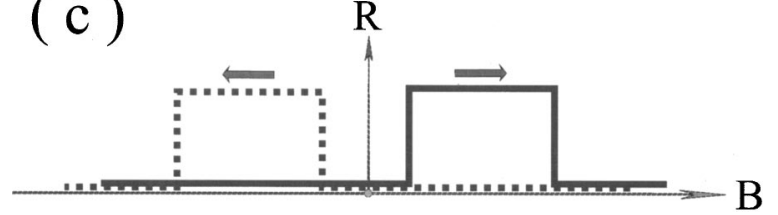

FIG. 1. (a) Simple device made of two FM electrodes connected by a conductor made of either metal or a 2DEG electrode. (b) If the two FM electrodes have different coercive fields, sweeping an external $B$ field switches their relative directions of magnetization between parallel and antiparallel configurations. (c) Expected magnetoresistance for such a device with a ballistic channel.

models emphasize one of the main advantages of 2DEG over a metal, namely, the larger electron mean free path that allows ballistic transport in the conducting channel. In the ballistic picture, spin injection across a heterojunction can be described by the transmission of spin-dependent Bloch waves from one electrode to the other. As shown in Fig. 2(a), in an all-metal junction, the Fermi energy $E_{F}^{L}$ and electron effective mass $m_{L}$ on the left FM electrode are comparable to those of the right metal electrode $E_{F}^{R}$ and $m_{R}$. Due to the spin-dependent density of states of the FM electrode which is characterized by its spin polarization, the number of Bloch waves transmitted across the interface is spin dependent. The spin injection rate $\eta$, defined as the normalized spindependent conductance, scales with the spin polarization in a simple picture. ${ }^{22}$ In the hybrid devices shown in Fig. 2(b), however, $E_{F}^{R}$ and $m_{R}$ of the 2DEG electrode are usually much smaller than $E_{F}^{L}$ and $m_{L}$ of the FM electrode. The 2DEG electrode allows therefore much fewer Bloch waves to be transmitted across the junction so that $\eta$ can no longer reflect the spin polarization nature of the FM electrode directly. Up to this point, ballistic pictures are consistent with the diffusive model of Schmidt et al. ${ }^{13}$ who first discovered the material mismatch problem and predicted a very small spin injection rate in typical hybrid devices. An important difference emerges by further studying the transmission probability of each Bloch wave across the junction, which is less than one in hybrid devices due to wave reflections at the interface. Such reflections are momentum dependent. This effect combined with the fact that the Fermi momentum of
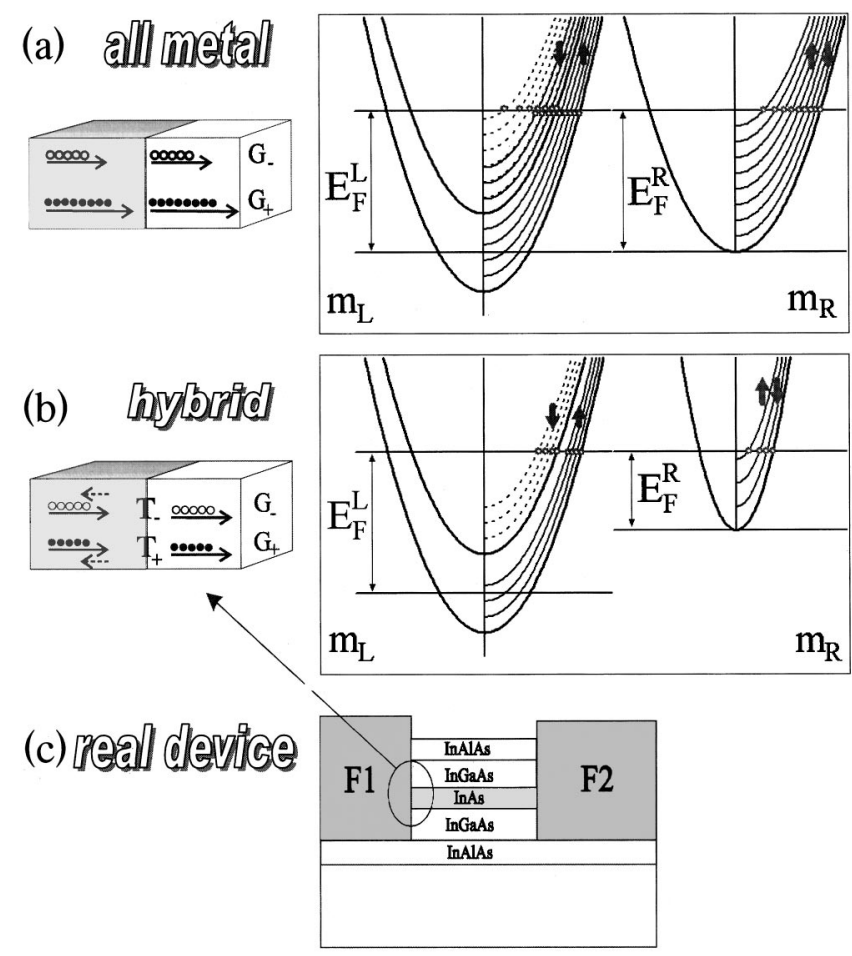

FIG. 2. Spin injection across a heterojunction made of (a) all-metal electrodes with matched Fermi energy $E_{F}$ and effective mass $m$ and (b) hybrid electrodes with mismatched band parameters. Spin-dependent conductance $G_{\sigma}$ in the hybrid device is determined by the spin-dependent transmission $T_{\sigma}$ of the Bloch waves at the interface. (c) Schematic of the cross section of the hybrid device used in the experiment.

the electrons in a FM electrode is spin dependent allows the spin injection rate $\eta$ across a hybrid junction to reflect indirectly the spin split nature of the FM electrode.

By analyzing effects of spin polarization, spin-orbit interaction, interface scattering, and band-structure mismatch on an equal quantum-mechanical footing, we recently obtained an analytical expression for the spin injection rate across a heterojunction. ${ }^{18}$ Figure 3 shows the dependence of the spin injection rate $\eta$ on the spin polarization $\eta_{0}$ of the injecting electrode. The solid line is calculated for matched electrodes with equal effective mass and Fermi energy in both electrodes that are used in the all-metal junction. It is consistent with an earlier result of a semiclassical model. ${ }^{22}$

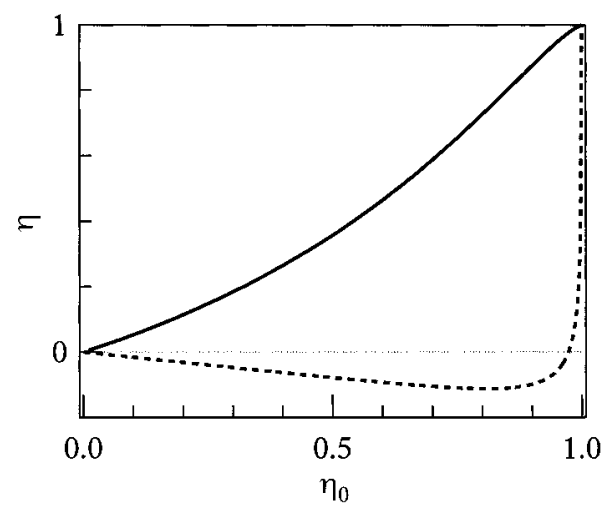

FIG. 3. Dependence of $\eta$ on spin polarization $\eta_{0}$ for an all-metal heterojunction (solid line) and a hybrid heterojunction (dashed line). 


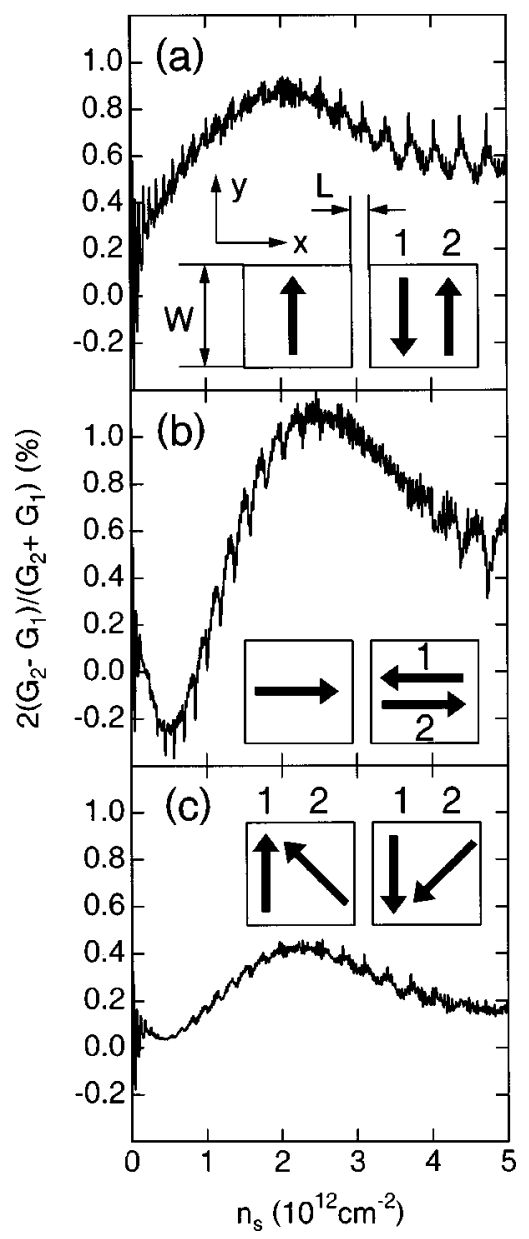

FIG. 4. Magnetoconductance ratios across a FM/2DEG/FM hybrid device. The curves are calculated for a channel length of $L=150 \mathrm{~nm}$ and a width of $W=1 \mu \mathrm{m}$.

The dashed line is calculated for mismatched electrodes assuming that the effective mass and Fermi energy of the right electrode are, respectively, $5 \%$ and $3 \%$ that in the left electrode. This is typical of hybrid junctions used in experiments. The significant difference in behavior reflects the characteristics between spin injection across an all-metal junction and one across a hybrid junction. In the all-metal junction, $\eta$ reflects the spin-dependent density of states at the Fermi level of the FM electrode, while in the hybrid junction $\eta$ is determined by the spin-dependent scattering of Bloch waves across an interface. Indeed, our result for mismatched electrodes shows generally similar behavior to that of the diffusive approach. ${ }^{13}$ However, by taking into account the intrinsic spin-dependent scattering of Bloch waves, the spin injection rate for a hybrid junction is found at a percentage level which is measurable. Benefiting from such a spindependent scattering nature, we found $\eta$ can be further enhanced by inserting a thin tunneling barrier at the interface. ${ }^{18}$ This is consistent with the result of an earlier diffusive model proposed by Rashba. ${ }^{14}$

To demonstrate the advantage of the semiconductor in a hybrid device, we extended our theory ${ }^{21}$ to calculate the change of magnetoconductance on carrier density for a realistic hybrid device, depicted in Fig. 2(c). Applying a gate voltage easily tunes the carrier density as well as the spin- orbit interaction of a 2DEG. Figures 4(a)-4(c) show the results for three different configurations of the ferromagnetic electrodes. In the spin-valve geometry depicted in Fig. 4(a) the background of the magnetoconductance ratio increases with the electron density at low densities and slightly decreases at higher ones. The latter is caused by a contribution by oblique modes. The coarse variation recovers the spinvalve characteristics of the ballistic spin filter transistor. ${ }^{16}$ In the case of the spin-transistor geometry shown in Fig. 4(b), modulation of the conductance ratio that changes its sign can clearly be observed. It is caused by the spin-precession state in the 2DEG and interference of spin eigenstates. ${ }^{3}$ The essential difference in this result to the large periodic modulation predicted by Datta and Das $^{3}$ is the reduced amplitude and deformed shape of the modulation, which is caused by the intrinsic nature of spin-dependent scattering of Bloch waves across a FM/2DEG interface. In the case of the mixed geometry in Fig. 4(c) the shape of the trace is similar to that in the spin-transistor geometry but the amplitude is further reduced by approximately a factor of 2 . All short-scale variations are due to Fábry-Perot type interference. Our results show the gating functionality of a hybrid device.

\section{EXPERIMENTAL APPROACH}

To measure the spin injection rate across a hybrid junction, we use a ferromagnetic permalloy $\left(\mathrm{Ni}_{40} \mathrm{Fe}_{60}\right)$ source (FM1) and drain (FM2) contacts deposited on a 2DEG confined in an InAs channel. ${ }^{12}$ The elastic mean free path of electrons in the 2DEG channel is $l_{e} \approx 1.3 \mu \mathrm{m}$. Figure 2(c) shows schematically the device structure. Four terminal resistance measurements were performed in a ${ }^{3} \mathrm{He}$ cryostat with a superconducting solenoid using standard ac lock-in techniques. A magnetic field $B$ was applied along the easy magnetization axis of the FM electrodes. Six samples with different combinations of the 2DEG channel length $L$ $(0.4 \mu \mathrm{m} \leqslant L<2 \mu \mathrm{m})$ and the ferromagnetic contact strip widths $F 1, F 2(0.5 \mu \mathrm{m} \leqslant F 1, F 2 \leqslant 3 \mu \mathrm{m})$ were measured in the temperature range from 0.3 to $10 \mathrm{~K}$. Five samples have channel width $W=2 \mu \mathrm{m}$, one has $W=4 \mu \mathrm{m}$. We find results that are reproducible in two senses: for different devices with the same channel length and for the same device measured in different cooling cycles.

Detailed magnetoresistance changes measured at different temperatures by sweeping the applied $B$ field over the range of $\pm 1000 \mathrm{G}$ have been reported elsewhere. ${ }^{12}$ In these experiments, since the coercive fields $B_{c 1}$ and $B_{c 2}$ of both contacts are smaller than $1000 \mathrm{G}$, the magnetization configuration of the $\mathrm{NiFe}$ contacts was switched between parallel and antiparallel configurations (Fig. 1(b)). Magnetoresistance changes in accordance with the simple picture shown in Fig. 1(c) (that shows) $\Delta R(B) / R_{0}=\left[R(B)-R_{0}\right] / R_{0}$ for the antiparallel configuration were observed on devices with a ballistic 2DEG channel with $L=0.45 \mu \mathrm{m}$. With an increase in temperature, the normalized resistance change at the peak position $\Delta R / R_{0}$ drops continuously from its low temperature maximum value of $0.2 \%$ and disappears for $T>10 \mathrm{~K}$. It is different from magnetoresistance features caused by the anisotropic magnetoresistance of permalloy electrodes or the 
( a )

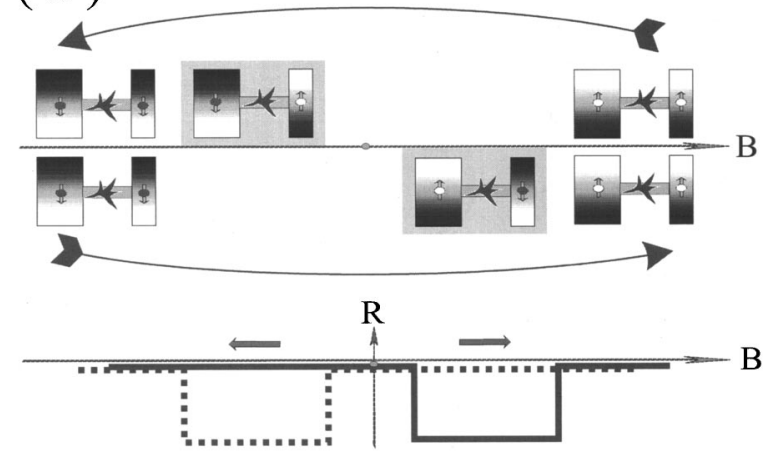

( b )

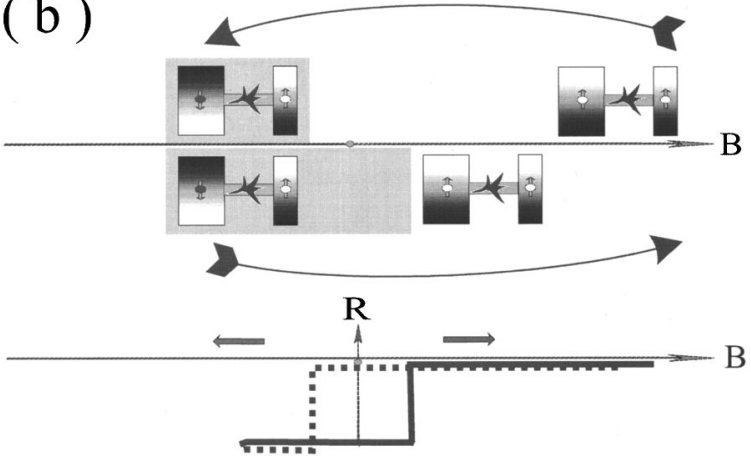

FIG. 5. (a) Hybrid devices with quasiballistic conducting channels showing negative magnetoresistance features. (b) By reversing the $B$ field after one of the FM electrodes is switched, a memory effect of the magnetoresistance is expected.

fringe field induced local Hall effect. Both effects are known to survive at high temperatures. ${ }^{23,24}$ From the normalized change in resistance at peak position $\Delta R / R_{0}$, we estimate polarization of $\eta \approx 4.5 \%$ for current through the interface. This value is of the same order as that calculated in our theory for spin injection. ${ }^{18}$

On devices with larger 2DEG channel lengths $L$, dips instead of peaks are observed. We found a systematic channel length dependence of the normalized resistance change ${ }^{12}$ that demonstrates the importance of transport in the 2DEG channel. Recently, two models were established. ${ }^{12,25}$ The first is based on combined effects of spin precession and scattering events that provides a qualitative explanation. ${ }^{12}$ The second ${ }^{25}$ explains the unexpected result as a combined effect of quantum coherence and spin-flip processes in mesoscopic disordered systems. Quantitative agreement with our data is found using the second model. Note that, in both models, the assumption of spin-polarized transport within the channel is essential, which confirms our observation of spin injection across the hybrid junction.

Based on these data, we illustrate in Fig. 5(a) the relationship between the magnetoresistance and the relative magnetization directions of the contacts for a hybrid device with a mesoscopic 2DEG channel. To further confirm this picture, we report here the results from a new experiment. The idea behind it is shown in Fig. 5(b). For a device with a quasiballistic 2DEG channel, if switching the direction of magnetization of both FM electrodes subsequently results in

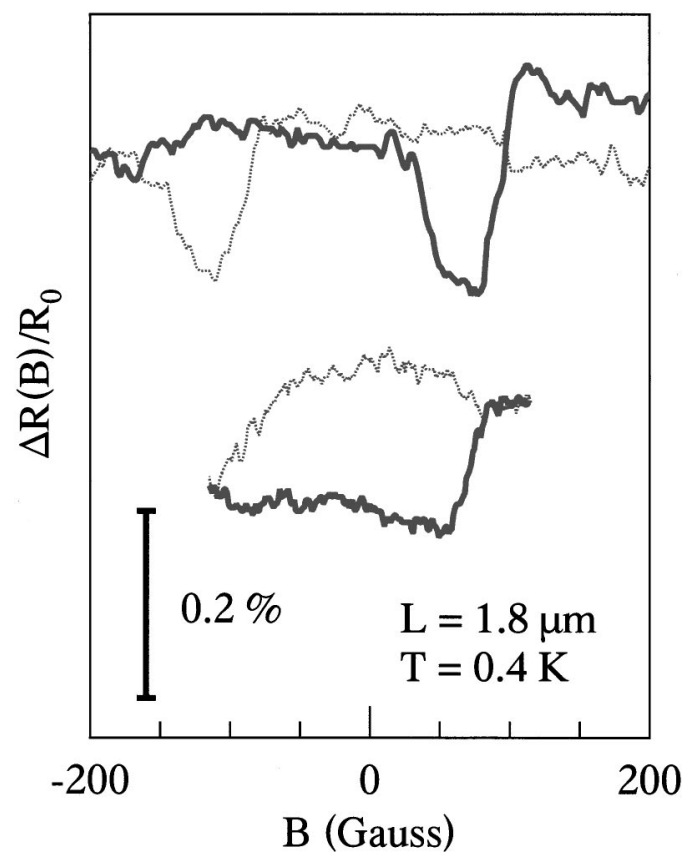

FIG. 6. (a) Negative magnetoresistance as well as a magnetoresistance loop that shows the memory effect measured for a hybrid device with a quasiballistic channel at $0.4 \mathrm{~K}$. The solid (dotted) lines correspond to the upward (downward) sweep direction of the $B$ field.

the negative magnetoresistance feature shown in Fig. 5(a), then by reversing the external magnetic field after one of the FM electrodes is switched, a MR structure, like that shown in Fig. 5(b), can be expected. This kind of experiment is also interesting in the sense that we can check whether a hybrid device can provide two stable resistance states in the absence of external magnetic field due to spin injection. We call it the memory effect of a hybrid device, whose source-drain resistance "remembers" the magnetization of one of the FM electrodes.

The measured magnetoresistance in a device with $L$ $=1.8 \mu \mathrm{m}$ and ferromagnetic contact strip widths $(F 1, F 2$ $=2,3 \mu \mathrm{m})$ at $0.4 \mathrm{~K}$ is plotted in Fig. 6. The upper trace is measured by sweeping the $B$ field between $\pm 1000 \mathrm{G}$, within this range both FM electrodes subsequently switch. The lower trace is measured under the same experimental conditions but with the $B$ field reversed at about $-100 \mathrm{G}$. A clear memory effect is observed. We would like to note that the dip structure observed in the upper trace is slightly noiser than the earlier measurement on the same device, ${ }^{12}$ however the main feature is well preserved, which demonstrates the reproducibility of our hybrid device. For a simple estimate, the difference between the two stable resistance states at $B$ $=0$ is proportional to $\eta^{2}$. The difference between the two stable resistance states at $B=0$ observed in our device is quite small due to the small spin injection rate $\eta$, but it is well defined compared with the noise level in our experiment. It demonstrates that the memory nature of the ferromagnet is well reserved in the hybrid device due to spin injection. We note that for hybrid devices with either a ballistic $^{18}$ or a diffusive $2 \mathrm{DEG}$ channel, ${ }^{14}$ theory predicts significant enhancement of $\eta$ by inserting a thin tunneling barrier at the interface. 


\section{CONCLUSION}

We demonstrated both theoretically and experimentally that spin injection across a hybrid FM/2DEG junction is characteristically different from that across the well known FM/M junctions. We show that the hybrid device keeps the gating functionality of the semiconductor. Magnetoresistance changes due to switching of the magnetization direction of the FM electrodes have been observed in hybrid devices. We find that spin injection allows the hybrid device to form two stable resistance states in the absence of an external $B$ field. It is a memory effect that is due to the nature of the ferromagnet electrode. We conclude that hybrid devices have the potential to combine functions of both the ferromagnet and the semiconductor. The main challenge is to enhance the spin injection rate.

\section{ACKNOWLEDGMENTS}

The authors thank M. Johnson, P. Streda, P. Seba, U. Rössler, D. Grundler, and G. Meier for valuable discussions. This work was supported financially by a NEDO International Joint Research Grant, the BMBF through Project No. 01BM905, and the DFG through Grant No. SFB 508. One of the authors (J.B.H.) thanks the European Commission for a grant.

\footnotetext{
${ }^{1}$ M. Johnson and R. H. Silsbee, Phys. Rev. Lett. 55, 1790 (1985).

${ }^{2}$ For a recent review, see, for example, P. Grünberg, Phys. Today 54, 31 (2001).

${ }^{3}$ S. Datta and B. Das, Appl. Phys. Lett. 56, 665 (1990).
}

${ }^{4}$ M. Oestreich, J. Hübner, D. Hägele, P. J. Klar, W. Heimbrodt, W. W. Rūhle, D. E. Ashenford, and B. Lunn, Appl. Phys. Lett. 747, 1251 (1999).

${ }^{5}$ H. X. Tang, F. G. Monzon, R. Lifshitz, M. C. Cross, and M. L. Roukes, Phys. Rev. B 61, 4437 (2000).

${ }^{6}$ G. Prinz, Phys. Today 48, 58 (1995); Science 282, 1660 (1998).

${ }^{7}$ F. G. Monzon and M. L. Roukes, J. Magn. Magn. Mater. 198-199, 632 (1999).

${ }^{8}$ S. Gardelis, C. G. Smith, C. H. W. Barnes, E. H. Linfield, and D. A. Ritchie, Phys. Rev. B 60, 7764 (1999).

${ }^{9}$ P. R. Hammar, B. R. Bennett, M. J. Yang, and M. Johnson, Phys. Rev. Lett. 83, 203 (1999); 84, 5024 (2000); Phys. Rev. B 61, 7202 (2000).

${ }^{10}$ A. T. Filip, B. H. Hoving, F. J. Jedema, B. J. van Wees, B. Dutta, and S. Borghs, Phys. Rev. B 62, 9996 (2000).

${ }^{11}$ G. Meier, T. Matsuyama, and U. Merkt, Phys. Rev. B (to be published).

${ }^{12}$ C.-M. Hu, J. Nitta, A. Jensen, J. B. Hansen, and H. Takayanagi, Phys. Rev. B 63, 125333 (2001).

${ }^{13}$ D. Ferrand, L. W. Molenkamp, A. T. Filip, and B. J. van Wees, Phys. Rev. B 62, R4790 (2000).

${ }^{14}$ E. I. Rashba, Phys. Rev. B 62, R16267 (2000).

${ }^{15}$ G. Kirczenow, Phys. Rev. B 63, 054422 (2001).

${ }^{16}$ D. Grundler, Phys. Rev. Lett. 86, 1058 (2001); Phys. Rev. B 63, 161307 (2001).

${ }^{17}$ R. H. Silsbee, Phys. Rev. B 63, 155305 (2001).

${ }^{18}$ C.-M. Hu and T. Matsuyama, Phys. Rev. Lett. 87, 066803 (2001).

${ }^{19}$ Th. Schäpers, J. Nitta, H. B. Heersche, and H. Takayanagi, Phys. Rev. B 64, 125314 (2001).

${ }^{20}$ L. W. Molenkamp, G. Schmidt, and G. E. W. Bauer, Phys. Rev. B 64, R121202 (2001).

${ }^{21}$ T. Matsuyama, C.-M. Hu, D. Grundler, G. Meier, and U. Merkt (unpublished).

${ }^{22}$ G. E. W. Bauer, Phys. Rev. Lett. 69, 1676 (1992).

${ }^{23}$ T. R. McGuire, and R. I. Potter, IEEE Trans. Magn. 11, 1018 (1975).

${ }^{24}$ G. Meier, Ph.D. thesis, University of Hamburg, Hamburg, Germany, 1999.

${ }^{25}$ P. Seba, P. Exner, K. N. Pichugin, A. Vyhnal, and P. Streda, Phys. Rev. Lett. 86, 1598 (2001). 